\title{
DESOSPITALIZAÇÃO EM CUIDADO PALIATIVOS: PERFIL DOS USUÁRIOS DE UMA UNIDADE NO RIO DE JANEIRO/ BRASIL*
}

\author{
Patrícia da Silva Olario1, Marléa Chagas Moreira², Isabel Borges Moreira², \\ José Carlos Amado Martins ${ }^{4}$, Alexandre Teófilo de Souza ${ }^{5}$
}

\begin{abstract}
RESUMO: Objetivo: conhecer o perfil dos usuários do serviço de desospitalização de uma unidade no Rio de Janeiro. Método: estudo descritivo e retrospectivo, com base no método de investigação quantitativa. Foram analisados prontuários de pessoas atendidas no período de janeiro de 2012 a dezembro de $2016(n=655)$. A amostra é constituída por $330(50,4 \%)$ usuários do sexo feminino, com 493 (75,3\%) acima de 60 anos. Maioritariamente 598 (91,3\%) residem na região metropolitana do RJ, 406 (61,9\%) têm câncer. Relativamente à demanda de cuidados técnicos prevalentes são as lesões de pele, 150 (22,9\%); a alimentação por via oral, 528 (80,6\%); e gástrica, 123 (18,7\%); estomias, 178 (27,1\%); e sonda vesical ou cistostomia $106(16,1 \%)$. Apresentavam dependência total, 424 (64,7\%). Conclusão: o perfil dos usuários é composto predominantemente por idosos, com dependência total e patologia oncológica requerendo implementação de estratégias que visem a condução destes casos.
\end{abstract}

DESCRITORES: Assistência domiciliar; Enfermagem oncológica; Cuidados de enfermagem; Cuidados Paliativos; Gestão em saúde.

\section{DEINSTITUTIONALIZATION IN PALLIATIVE CARE: PROFILE OF USERS OF A UNIT IN RIO DE JANEIRO/BRAZIL}

ABSTRACT: Objective: Gain knowledge on the profile of users of a residential care service provided by a hospital in Rio de Janeiro as part of a deinstitutionalization strategy for individuals in palliative care. Method: descriptive and retrospective study based on quantitative investigation. The medical records of patients who received care at a home care service from January 2012 to December $2016(n=655)$ were analyzed. Of these, $330(50.4 \%)$ were women and $493(75.3 \%)$ were older than 60 years. Most of them, $598(91.3 \%)$ lived in the Metropolitan region of Rio de Janeiro and 406 (61.9\%) have cancer. Regarding the demand for technical care, there was a prevalence of care related to skin lesions, 150 (22.9\%); followed by oral feeding, $528(80.6 \%)$; and gastrostomy, $123(18.7 \%)$; ostomies, $178(27.1 \%)$ and bladder catheter or cystostomy, $106(16.1 \%)$. In addition, $424(64.7 \%)$ users were entirely dependent on care. Conclusion: Regarding the profile of the users, most of them were elderly individuals entirely dependent on care and diagnosed with cancer. Strategies should be implemented for the management of these individuals.

KEYWORDS: Home care; Oncological nursing; Nursing care; Palliative care; Health management.

\section{DESHOSPITALIZACIÓN EN CUIDADOS PALIATIVOS: PERFIL DE LOS USUARIOS DE UNA UNIDAD EN RIO DE JANEIRO/BRASIL}

RESUMEN: Objetivo: conocer el perfil de los usuarios del servicio de deshospitalización de una unidad en Rio de Janeiro. Método: estudio descriptivo y retrospectivo, con base en el método de investigación cuantitativa. Se analizaron prontuarios de personas atendidas en el periodo de enero de 2012 a diciembre de $2016(n=655)$. La muestra tiene $330(50,4 \%)$ usuarios del sexo femenino, con 493 (75,3\%) mayores de 60 años. La mayoría $598(91,3 \%)$ viven en la región metropolitana de RJ, $406(61,9 \%)$ tienen cáncer. Acerca de la demanda de cuidados técnicos, son prevalentes las lesiones de piel, 150 22,9\%); la alimentación por vía oral, 528 (80,6\%); y gástrica, 123 (18,7\%); ostomías, 178 (27,1\%); y sonda vesical o cistostomía 106 (16,1\%). Presentaban dependencia total 424 (64,7\%). Conclusión: el perfil de los usuarios fue predominantemente de ancianos, con dependencia total y patología oncológica, siendo necesaria implementación de estrategias para el acompañamiento de estos casos.

DESCRIPTORES: Asistencia domiciliar; Enfermería oncológica; Cuidados de enfermería; Cuidados paliativos; Gestión en salud.

*Artigo vinculado a tese de doutorado intitulada: Desospitalização em cuidados paliativos oncológicos: reconfiguração do processo assistencial para a atuação multiprofissional, Rio de Janeiro, 2018.

${ }^{1}$ Enfermeira. Doutoranda em Enfermagem. Coordenadora do Serviço de Atenção à Desospitalização do Hospital Federal Cardoso Fontes/ Ministério da Saúde. Rio de Janeiro, RJ, Brasil.

${ }^{2}$ Enfermeira. Doutora em Enfermagem. Docente do Departamento de Metodologia da Enfermagem da Escola de Enfermagem Anna Nery/ Universidade Federal do Rio de Janeiro. Rio de Janeiro, RJ, Brasil.

${ }^{3}$ Enfermeira. Doutora em Enfermagem. Professora Coordenadora e Docente da Unidade Científico-pedagógica de Enfermagem Médico-cirúrgica da Escola Superior de Enfermagem de Coimbra. Coimbra, Portugal.

${ }^{4}$ Enfermeiro. Pós-doutor em Enfermagem. Professor Coordenador e Docente da Unidade Científico-pedagógica de Enfermagem Médico-cirúrgica da Escola Superior de Enfermagem de Coimbra. Coimbra, Portugal.

${ }^{5}$ Administrador. Especialista em Gestão em Administração Pública. Coordenador Substituto do Serviço de Atenção à Desospitalização do Hospital Federal Cardoso Fontes/ Ministério da Saúde. Rio de Janeiro, RJ, Brasil. 


\section{INTRODUÇÃO}

A atualidade desta temática desvela-se na crescente demanda de pessoas em cuidados paliativos, sobretudo na área oncológica, levando à reflexão acerca da necessidade de "novos arranjos tecnológicos"(1) para a promoção do cuidar, visando à articulação em rede e continuidade dos cuidados de saúde.

De acordo com dados da Organização Mundial de Saúde (OMS) sobre a situação da oferta de cuidados paliativos no contexto internacional, o câncer aparece como uma das principais doenças crônicas que permeiam este universo, com a estimativa para o ano de 2030 de 27 milhões de novos casos, 17 milhões de óbitos e 75 milhões de pessoas vivendo anualmente com a patologia oncológica, o que demonstram a necessidade de articulações e investimentos em saúde visando contemplar a esta demanda ${ }^{(2-3)}$.

O modelo assistencial terá de acompanhar as mudanças do perfil epidemiológico populacional, representado pela alta incidência de doenças crônico-degenerativas, em especial das patologias oncológicas, com necessidades de cuidados paliativos em virtude de um envelhecimento populacional e da mudança de estilos de vida ${ }^{(4)}$.

Neste sentido, o Ministério da Saúde brasileiro vem investindo em políticas e estratégias paraminimizar os impactos sociais e decorrentes da magnitude das situações crônicas de saúde, promovendo a qualidade de vida das pessoas nessas condições, bem como a integração e participação da família neste cenário, proporcionando o cuidar em outros ambientes que não exclusivamente o hospitalar.

O termo "desospitalização"(5) se refere à desinstitucionalização de pessoas internadas em ambiente hospitalar, favorecendo a agilidade no processo de alta para o domicílio e reinserção da pessoa na rede, considerando os princípios da humanização, continuidade do cuidado e participação da família. E considerando um dos "eixos centrais do atendimento domiciliar".

A atenção domiciliar é uma das formas possíveis de prestar cuidadosà pessoa com câncer em cuidados paliativos e seus familiares, com a otimização do processo de transição de cuidados, como previsto nas políticas públicas brasileiras e internacionais envolvidas neste contexto, bem como no cenário internacional, possibilitando a articulação com as diferentes instâncias que compõe a rede de atenção à saúde, buscando deste modo estratégias para solucionar as lacunas no atendimento às demandas de cuidados da pessoa e da família, de modo integrado e multidisciplinar ${ }^{(6-10)}$.

Considerando os cuidados paliativos como "dispositivo biopolítico criado em defesa da sociedade", é pertinente o incentivo de modelos de atenção previstos e possíveis que já são implementados mundialmente e que ainda manifestam-se nos países em desenvolvimento de modo incipiente, no que tange às articulações e ao desenvolvimento de ações integradas, tais como: atendimento domiciliar, hospices e serviços de atenção oncológicas ${ }^{(11-12)}$.

A busca pela assistência hospitalar, não considerando outras possibilidades de modelos de atenção, traduz-se em uma tentativa de resolução curativa da doença. Porém, diante da impossibilidade desta, a articulação em rede de atenção à saúde para a manutenção do cuidado desta pessoa ainda se apresenta como uma lacuna, sendo necessário repensar alternativas, tais como as medidas de desospitalização de forma integrada, centradas nas necessidades da pessoa com câncer e da família em sua multidimensionalidade.

Assim, desospitalizar está para além da simples retirada da pessoa do hospital, sendo um movimento compreendido como um ato de continuidade das ações iniciadas no ambiente intra-hospitalar, bem como a manutenção da articulação em rede, de forma integrada e humana.

Deste modo, buscando a valorização da desospitalização no cenário dos cuidados paliativos oncológicos no Brasil, como uma estratégia de atenção à saúde, objetivou-se neste estudo conhecer o perfil dos usuários do serviço de desospitalização do Hospital Federal Cardoso Fontes no Rio de Janeiro/ Brasil. 


\section{MÉTODO}

Este estudo faz parte de uma pesquisa (tese), que trata do processo de desospitalização da pessoa com câncer em cuidados paliativos, na perspectiva da equipe e unidade familiar. O desenho metodológico desta pesquisa está amparado em um estudo descritivo e retrospectivo, com base nos pressupostos do método de investigação quantitativa.

Foi realizado levantamento documental (prontuários) no Hospital Federal Cardoso Fontes (HFCF) no bairro de Jacarepaguá, no município do Rio de Janeiro/ Brasil, em um serviço de atenção à desospitalização (SADES), que atende pessoas em cuidados paliativos no processo de transição do hospital para o domicílio, com atendimento e tratamento nesta unidade hospitalar.

Para a efetivação da coleta de dados, no período de janeiro a março de 2017, foram realizados os levantamentos dos prontuários de pessoas atendidas pelo serviço no período de janeiro de 2012 a dezembro de 2016 ( $n=655$ ). Como critérios de inclusão consideraram-se os prontuários de pessoas acompanhadas a partir do processo de alta hospitalar até o seu cuidado no domicílio, com termo de consentimento assinado por um familiar, responsabilizando-se como cuidador formal da pessoa em questão, no ato do acolhimento no serviço. Como critérios de exclusão consideraram-se às solicitações de parecer para o serviço, sem a efetivação da implementação do processo de desospitalização.

Os dados obtidos foram codificados e digitalizados em planilha eletrônica e posteriormente analisados através do auxílio do software SPSS versão 21.0 ${ }^{\circledR}$ (SPSS for Windows, Chicago, EUA), sendo tratados com estatística de modo descritivo e apresentados em tabelas de frequência com valores absolutos (n) e percentuais (\%) para as variáveis categóricas.

A pesquisa foi aprovada pelo Comitê de Ética em Pesquisa da Escola de Enfermagem Anna Nery/UFRJ e autorizada pela instituição coparticipante, sob o Parecer n. $\square$ 1.548.669. No que tange aos aspectos éticos, foram observadas questões envolvendo seres humanos de acordo com a Resolução n $\square$. 466/2012 do Conselho Nacional de Saúde do Ministério da Saúde ${ }^{(13)}$.

\section{RESUlTADOS}

A amostra do estudo constitui-se de 655 pessoas em cuidados paliativos, cadastradas no serviço no período estudado. A Tabela 1 descreve a distribuição sociodemográfica que constavam no prontuário como usuários do serviço.

Observou-se que com relação ao cenário estudado, não existe divergência de gênero na amostra, considerando que o sexo feminino é constituído por 330 pessoas $(50,4 \%)$ e o sexo masculino por $325(49,6 \%)$, o que não é relevante estatisticamente. Com relação à faixa etária predominante, esta é composta por pessoas acima de 60 anos em 493 usuários (75,3\%), com composição familiar de 4 a 5 membros em $292(44,6 \%)$ dos usuários. A região predominante de moradia destas pessoas encontrase localizada na área metropolitana do Rio de Janeiro com 598 (91,3\%) dos usuários distribuídos em diversos bairros, porém, 57 (8,7\%) residem em outros municípios fora do Rio de Janeiro. 
Tabela 1 - Distribuição dos usuários do SADES, segundo aspectos sociodemográficos. Rio de Janeiro, RJ, Brasil, 2017

\begin{tabular}{cccc}
\hline Variáveis & Categorias & $\mathbf{n}$ & $\mathbf{\%}$ \\
\hline Sexo & Feminino & 330 & 50,4 \\
& Masculino & 325 & 49,6 \\
\hline \multirow{3}{*}{ Faixa Etária } & $\leq 18$ anos & 02 & 0,3 \\
& $19-29$ anos & 07 & 1,1 \\
& $30-39$ anos & 26 & 4 \\
& $40-49$ anos & 50 & 7,6 \\
Composição familiar & $50-59$ anos & 77 & 11,7 \\
& $\geq 60$ anos & 493 & 75,3 \\
\hline \multirow{2}{*}{ Região do RJ em que reside } & $2-3$ membros & 198 & 30,2 \\
& $4-5$ membros & 292 & 44,6 \\
& $6-7$ membros & 137 & 20,9 \\
& Mais que 7 membros & 28 & 4,3 \\
\hline
\end{tabular}

A Tabela 2 apresenta a distribuição dos diagnósticos dos usuários atendidos pelo serviço no período de 2012 a 2016. Com relação à tipologia dos diagnósticos, a maioria tem câncer, 406 (61,9\%), com predominância no câncer de próstata, 117 (17,9\%), seguido pelo câncer de mama presente em 98 (15\%) dos casos. Nos outros diagnósticos não oncológicos, estes perfazem um total de 249 (38,1\%) dos casos com predomínio dos casos de Alzhiemer 109 (16,6\%).

Tabela 2 - Distribuição dos diagnósticos dos usuários atendidos no SADES/HFCF. Rio de Janeiro, RJ, Brasil, 2017

\begin{tabular}{|c|c|c|c|}
\hline Variáveis & Categorias & $\mathbf{n}$ & $\%$ \\
\hline \multirow[t]{2}{*}{ Tipos de diagnósticos } & Câncer & 406 & 61,9 \\
\hline & Outros diagnósticos & 249 & 38,1 \\
\hline \multirow{20}{*}{$\begin{array}{l}\text { Diagnóstico } \\
\text { Principal }\end{array}$} & Câncer de próstata & 117 & 17,9 \\
\hline & Câncer de mama & 98 & 15 \\
\hline & Câncer de pulmão & 31 & 4,7 \\
\hline & Câncer de colo uterino & 25 & 3,8 \\
\hline & Câncer de vulva & 10 & 1,5 \\
\hline & Câncer de estômago & 46 & 7 \\
\hline & Alzheimer & 109 & 16,6 \\
\hline & $\mathrm{HIV}$ & 2 & 0,3 \\
\hline & Sequelas de AVC/AVE & 99 & 15,1 \\
\hline & Câncer renal & 20 & 3,1 \\
\hline & Câncer de reto/intestino & 35 & 5,3 \\
\hline & Câncer de pâncreas & 1 & 0,2 \\
\hline & Câncer de laringe & 2 & 0,3 \\
\hline & Câncer de canal anal & 2 & 0,3 \\
\hline & Alterações neurológicas diversas & 39 & 6 \\
\hline & Câncer de ovário & 4 & 0,6 \\
\hline & Linfoma de hodking & 7 & 1,1 \\
\hline & Linfoma não hodking & 1 & 0,2 \\
\hline & Câncer cerebral & 3 & 0,5 \\
\hline & Câncer de tireoide & 4 & 0,8 \\
\hline
\end{tabular}


A Tabela 3 apresenta a distribuição do fluxo dos usuários pós-desospitalização ocorridos no período de 2012 a 2106 . As articulações em rede com encaminhamentos para a rede básica de atenção totalizaram $221(33,8 \%)$ e os óbitos $337(51,4 \%)$. Na distribuição de encaminhamentos/ano observou-se que no ano de 2012 nenhum encaminhamento foi realizado, sendo 12 (1,8\%), em 2013, 26 (4\%) em 2014, em 2015 ocorreram 117 (17,9\%)e em 2016 registrou-se 66 (10,1\%) encaminhamentos.

No que tange aos óbitos/ano, em 2012 ocorreram 66 (10,1\%), em 2013 foram 48 (7,3\%), já no ano de 2014 os óbitos compuseram 32 (4,9\%), em 2015 totalizaram 35 (5,3\%) e em 2016 foram 156 (23,8\%). Com relação à reinternação hospitalar, esta ocorreu em 24 (3,7\%) dos casos.

Tabela 3 - Distribuição do fluxo dos usuários do SADES/HFCF pós-desospitalização. Rio de Janeiro, RJ, Brasil, 2017

\begin{tabular}{lccc}
\hline Variáveis & Categorias & $\mathbf{n}$ & $\%$ \\
\hline Total de encaminhamentos para atenção primária & & 221 & 33,8 \\
\hline Encaminhamentos para atenção primária/ano & 2013 & 12 & 1,8 \\
& 2014 & 26 & 4 \\
& 2015 & 117 & 17,9 \\
& 2016 & 66 & 10,1 \\
\hline Total de óbitos & & 337 & 51,4 \\
\hline \multirow{2}{*}{ Óbitos / ano } & 2012 & 66 & 10,1 \\
& 2013 & 48 & 7,3 \\
& 2014 & 32 & 5,9 \\
& 2015 & 35 & 23,3 \\
\hline Reinternação hospitalar & 2016 & 156 & 3,7 \\
& Sim & 24 & 96,3 \\
\hline
\end{tabular}

*A tabela em questão não totaliza 100\% em virtude dos usuários que permaneceram ativos no serviço no ano de 2016 que equivalem a $n=97(14,8 \%)$

A Tabela 4 apresenta a distribuição da demanda dos cuidados, terapêutica utilizada no domicílio e nível de dependência de cuidados das pessoas atendidas no serviço. As lesões de pele estavam presentes em $150(22,9 \%)$ dos casos. Com relação à tipologia de demanda de cuidados, é possível observar que referente à alimentação $528(80,6 \%)$ alimentavam-se por via oral, $123(18,7 \%)$ por via gastrostomia e quatro $(0,6 \%)$ por via sonda nasoenteral.

No que tange à utilização de estomias, esta foi identificada em $178(27,1 \%)$ dos casos, sendo 123 $(18,7 \%)$ gastrostomias, $18(2,7 \%)$ traqueostomias, e 37 (5,6\%) outras estomias (ileostomia, jejunostomia, colostomia).

Os que utilizaram oxigenoterapia no período representaram 28 (4,3\%). Os que utilizavam sonda vesical de demora ou cistostomia totalizavam 106(16,1\%). As terapêuticas utilizadas por via oral ou via gastrostomia representavam $630(96,2 \%)$, injetáveis $13(2 \%)$, soroterapia 3(0,5\%), hipodérmica 8 $(1,2 \%)$, e transdérmica1 $(0,1 \%)$. Com relação ao nível de dependência de cuidados, a maioria, 424 $(64,7 \%)$, apresenta nível de dependência total, enquanto $170(26 \%)$ apresentam nível de dependência parcial e apenas 61 (9,3\%) mostravam-se independentes durante o processo de desospitalização. 
Tabela 4 - Distribuição de demanda de cuidados técnicos, nível de dependência de cuidados e terapêutica utilizada pelos usuários do SADES/HFCF no domicílio. Rio de Janeiro, RJ, Brasil, 2017

\begin{tabular}{clcc}
\hline Variáveis & \multicolumn{1}{c}{ Categorias } & $\mathbf{n}$ & $\mathbf{\%}$ \\
\hline & Lesões de pele & 150 & 22,9 \\
& Alimentação Oral & 528 & 80,6 \\
& Alimentação via Gastrostomia & 123 & 18,7 \\
& Alimentação via Sonda nasoenteral & 4 & 0,6 \\
Demanda & Ostomias & 178 & 27,1 \\
decuidados & Gastrostomia & 123 & 18,7 \\
técnicos & Traqueostomia & 18 & 2,7 \\
& Outras ostomias (jejunostomiaileostomiacistostomia, colostomia) & 37 & 5,6 \\
& Oxigenoterapia & 28 & 4,3 \\
& Sonda vesical de demora/ cistostomia & 106 & 16,1 \\
\hline & Via oral ou via Gastrostomia & 630 & 96,2 \\
& Via injetável & 13 & 2 \\
Terapêutica & Soroterapia & 3 & 0,5 \\
utilizada no & Via hipodérmica & 8 & 1,2 \\
domicílio & Via transdérmica & 1 & 0,1 \\
\hline Nível de & Total & 424 & 64,7 \\
dependência & Parcial & 170 & 26 \\
& Independente & 61 & 9,3 \\
\hline
\end{tabular}

\section{DISCUSSÃO}

Os dados sociodemográficos indicam o perfil dos usuários do Serviço de Atenção à Desospitalização (SADES) do HFCF, composto por 50,4\% de população feminina e 46,9\% de população masculina, não existindo divergência de gênero na amostra, em virtude da diferença nos dados, o que não é relevante estatisticamente. Acredita-se, ainda, que tais dados se justificam no cenário estudado, em virtude dos serviços de urologia e ginecologia especializados no atendimento das doenças oncológicas com relevância na rede de atenção à saúde, o que nos permite observar que não existe um gênero predominante na pesquisa.

Outro ponto a ser destacado na observação dos dados está nos moradores fora da Região Metropolitana do Rio de Janeiro $(8,7 \%)$, que mesmo residindo em outros municípios recebem o atendimento pela instituição estudada, o que pode caracterizar um itinerário terapêutico na busca por atendimento especializado, sobretudo na atenção oncológica ${ }^{(14)}$.

Acredita-se que este deslocamento pela busca de assistência possa estar atrelado à dificuldade de atendimento e diagnóstico precoce para os casos de câncer nos municípios menores, o que provoca sobrecarga nos hospitais especializados dos grandes centros urbanos, bem como a possível demora na continuidade do tratamento e maior dificuldade de articulação em rede.

Assim, observa-se à relevância da articulação do serviço de desospitalização com a atenção primária, favorecendo os encaminhamentos e acolhimentos $(33,8 \%)$ de pessoas, mesmo diante de um percentual elevado de óbitos $(51,4 \%)$ que muitas vezes caracteriza um diagnóstico tardio ou itinerário terapêutico sem resolutividade do caso.

Outro ponto relevante pauta-se no baixo índice de reinternação hospitalar (3,7\%), o que pode estar vinculado ao número de óbitos ou à implementação de ações integradas em saúde, que podem e devem ser utilizadas, dentre outros aspectos, como um mecanismo gerencial na otimização de leitos hospitalares.

Nesta perspectiva, a articulação e integração das diferentes esferas que compõe o sistema de saúde no Brasil, apresentam-se como uma alternativa para a crescente demanda das pessoas em cuidados 
paliativos, sobretudo os com patologias oncológicas, possibilitando o cuidado extra-hospitalar, com a participação de todos os envolvidosna condução e gerenciamento do cuidado.

Outro aspecto identificado encontra-se na predominância da composição familiar entre 4 e 5 membros, que contribui como um facilitador do processo de desospitalização, considerando a possibilidade do treinamento e participação de mais de um membro na execução do cuidar, construindo deste modo uma estrutura de auxílio mútuo na unidade familiar.

Como evidencia um estudo desenvolvido no âmbito da construção de competências dos cuidadores de doentes com câncer, uma das formas encontradas por eles para assegurar o cuidado no domicilio se refere à integração dos membros da família, nuclear e alargada, criando-se assim um padrão de cuidados, em que a extensão do envolvimento de outros elementos dependia da disponibilidade de tempo dos mesmos e dos seus recursos pessoais ${ }^{(15)}$.

Sem dúvida que a família constitui recurso inestimável no processo de cuidados à pessoa com câncer, não só pelo valor afetivo que os cuidados desenvolvidos por esta unidade têm para com esta pessoa, mas também pelo valor econômico que este trabalho invisível tem para a sociedade ${ }^{(16)}$.

Vivemos num tempo em que os sistemas de saúde necessitam de desenvolvimento de táticas para se tornarem mais sustentáveis, e os cuidadores - desde que devidamente apoiados - podem constituir este valoroso recurso.

Assim, pensar no sistema de saúde é vislumbrar a necessidade de criação de uma política de cuidados que promova a retroalimentacão entre o cuidado formal e o informal, garantindo apoio ao cuidador e maior interação entre as diferentes esferas.

Considerando o perfil dos usuários do Serviço de Atenção à Desospitalização, que apresenta um percentual de mulheres acometidas por câncer de mama, colo uterino, vulva e outras patologias com perfil para o tratamento paliativo, é importante refletirmos acerca do papel destas mulheres como representantes do cuidado informal, considerando a função que estas exercem na sociedade, e que quando debilitadas por uma patologia grave acabam por desestruturar a família e seus pares na organização do contexto e dinâmica diária.

Um estudo fenomenológico recente, ${ }^{(17)}$ realizado em Portugal, mostrou idênticos resultados, apresentando cuidadores maioritariamente mulheres, com idade superior a 65 anos, apresentando várias comorbidades e referindo dificuldades e sentimentos negativos associados ao processo de cuidado informal.

Deste modo, é premente que os dois sistemas de cuidados (formal e informal) trabalhem em conjunto de forma a assegurar o bem-estar da pessoa dependente e dos cuidadores informais ${ }^{(15-16)}$.

As necessidades de cuidados técnicos, apresentadas nos dados deste estudo, demonstram que não há impossibilidade para a alta hospitalar em virtude dessa demanda, podendo ser adotadas medidas de desospitalização para a implementação do cuidado com o treinamento e participação do cuidador, visando à manutenção das condições de saúde no domicílio e prevenção de internação hospitalar com uso de tecnologias duras desnecessárias.

Para o desenvolvimento destas condutas, torna-se imperiosa a conscientização de equipes intra e extra-hospitalares acerca dos conceitos que permeiam a paliação, bem como a condução eficaz deste processo no que tange a todas as esferas que gerenciam este cuidar.

Nesta ótica, a desospitalização deverá ser vislumbrada antecedendo o período de alta hospitalar, com diálogos Inter equipes e familiares, visando aos treinamentos necessários e à sua manutenção no domicílio, possibilitando a troca de informações, infraestrutura adequada para a otimização do processo de cuidar, com garantia de acesso e segurança, esclarecimentos de dúvidas e detecção precoce de complicações ${ }^{(12)}$.

Com relação ao número de casos de pessoas com câncer atendidas pelo serviço estudado (61,9\%) que se sobrepõe aos outros diagnósticos $(38,1 \%)$, observamos um alinhamento comas estimativas de pessoas vivendo com câncer e em cuidados paliativos no futuro, em especial no Brasil com estimativa de 600 mil novos casos para os anos de 2016 e $2017^{(18)}$. 
O predomínio dos índices de casos de câncer de próstata (17,9\%) e de mama (15\%) neste cenário da pesquisa leva-nos a pensar na necessidade de construção de uma rede de cuidados oncológicos, tendo em vista as estimativas desta patologia e os altos índices destes tipos de cânceres, sobretudo em idosos no Brasil e no Rio de Janeiro(19).

Ainda, o número de doentes com câncer de próstata no serviço estudado $(17,9 \%)$ demonstra uma correlação direta com a demanda de cuidados apresentadas, no que tange à utilização de sonda vesical ou cistostomia $(16,1 \%)$, necessitando de trocas periódicas no domicílio e orientações específicas, o que torna a desospitalização indispensável na condução destes processos, prevenção de complicações e hospitalizações desnecessárias.

Assim, a implementação de ações de desospitalizar que possibilitem a participação da família nos cuidados, a independência do usuário, a rotatividade de leitos hospitalares e um menor tempo de internação são fundamentais; articulando-se equipes da atenção básica, equipes hospitalares e equipes de desospitalização no planejamento e execução de atividades coordenadas e integradas de saúde, visando à promoção da melhoria do atendimento prestado, à humanização da assistência e à otimização de leitos hospitalares ${ }^{(5)}$.

Neste sentido, acredita-se que os encaminhamentos para a atenção primária, garantindo o suporte necessário de referência e contra referência para orientações e atendimentos de rotina, em casos de situações estáveis e com menor demanda de cuidados técnicos, proporcionando a base para lidar com questões subjetivas decorrentes das relações de afeto, doença avançada ou proximidade da morte sejam indispensáveis ${ }^{(5)}$.

Outro ponto relevante encontra-se nos apontamentos apresentados por alguns autores acerca do envelhecimento populacional, como sendo uma das principais causas das doenças crônico degenerativas, ${ }^{(19-21)}$ o que dialoga com os dados encontrados neste estudo que demonstra o predomínio da população com mais de 60 anos, o que nos leva a refletir sobre a necessidade de modificações no gerenciamento e implementação dos cuidados para esta população, considerando o crescente aumento desta demanda.

As diversas implicações que influenciam o processo de saúde e doença, em especial nos cuidados paliativos, refletem a busca por um cuidado que venha abarcar aspectos que vão para além do fisiológico e que contemplem a qualidade de vida e redução do sofrimento, visando o ser humano no que tange a um misto de diversos fatores, que resultam na sua concepção de mundo, e atitudes frente aos desafios apresentados.

Avanços no cuidar em domicílio, ainda precisam ser alcançados, sobretudo no que se refere ao controle da dor e adoção de outras vias de administração de medicamentos que não apenas a oral ou gástrica, devendo ser repensada e mais utilizada outras vias para terapêutica medicamentosa, em especial em se tratando de analgesia para as pessoas com dor crônica, podendo ser utilizada a via hipodérmica ou transdérmica visando a uma maior eficácia e conforto(22).

Deste modo, conhecer um serviço de desospitalização nos possibilita refletir sobre novas formas de cuidar em ambientes que ultrapassem os muros hospitalares, nos permite reconhecer a necessidade de diálogos compartilhados com troca de saberes, visando a crescente demanda de doenças crônicas, sobretudo as oncológicas e em cuidados paliativos.

Ademais, este perfil demonstra a necessidade de discussões sobre as alternativas de cuidar, tais como a desospitalização que exige a construção de estratégias conjuntas, visando à manutenção da continuidade deste processo em domicílio.

Ainda, penso serem necessárias discussões mais ampliadas que abarquem o estreitamento das instâncias que compõe a Rede de Atenção à Saúde, com a elaboração de um planejamento conjunto, com definição de metas, visando a uma melhoria das ações no processo de desospitalizar, nas questões de desmistificar o cuidar oncológico em ambientes extra-hospitalares.

Garantir a manutenção de cuidados extra-hospitalares, com baixo índice de reinternação em pessoas com doenças oncológicas, reafirma o direito de escolher o local de tratamento, bem como proporcionar uma morte mais digna e com livre escolha junto à pessoa/família e equipe, discutindo riscos e benefícios e tornando a participação fundamental no processo de gerenciamento do cuidado. 
Possibilitar a discussão dos anseios, medos e inseguranças que permeiam todo o universo do cuidado oncológico no processo de desospitalização, exige sensibilidade em transmitir confiança, auxiliar na construção do cuidado em um ambiente diferente do hospitalar e favorecer as adaptações necessárias à manutenção do cuidado.

Conhecer o perfil de um serviço favorece, dentre outros aspectos, na elaboração e condução de cuidados voltados a uma determinada população com as características mapeadas, devendo estas ser adequadas às necessidades de cada pessoa e família durante as discussões que antecedem a alta hospitalar.

Continua a ser importante estudar estas temáticas que abordam os cuidados de pessoas com patologias oncológicas, sobretudo em cuidados paliativos, com profundidade. Em Portugal, o estudo da temática dos cuidados paliativos em dissertações de mestrado e teses de doutorado tem uma constante crescente, mas afirmam os autores que desenvolveram o estudo que aponta estes dados ${ }^{(23)}$ que há ainda várias lacunas por preencher, o que também representa a realidade brasileira.

\section{- CONCLUSÃO}

Com este estudo é possível concluir que o perfil dos usuários deste serviço demonstra a necessidade de busca por estratégias que possam colaborar com os cuidados paliativos, em diferentes cenários, visando otimizar às ações integradas e em rede de cuidados.

Para tal, a formação em cuidados paliativos para todas as equipes da rede de atenção à saúde, em virtude da crescente demanda, torna-se a cada dia mais imperiosa, devendo ser implementada em diferentes níveis com vistas aos diferentes níveis de complexidade destes cenários.

Ao adentrarmos no domicílio com a pessoa em cuidados paliativos trazemos à tona os anseios, as inseguranças, os medos que norteiam o processo de cuidar, tornando o gerenciamento deste processo algo fundamental, de modo a minimizar tais fatores, colaborando para que a pessoa e família se tornem também agentes e participantes deste contexto, possibilitando deste modo o sucesso das ações planejadas, das articulações em rede e menor taxa de reinternação hospitalar possível.

Diante de tal fato, acredita-se que a necessidade de reflexão por parte dos profissionais, visando à implementação e gerenciamento de cuidados à pessoa em cuidados paliativos no processo de desospitalização, seja primordial, tendo em vista a previsão de crescimento desta demanda e das políticas vigentes, que vem sendo discutidas e implementadas mundialmente.

Ainda, acredita-se que esta pesquisa possa incentivar a constituição de novos estudos pautados em aspectos que permeiam a desospitalização da pessoa com câncer em cuidados paliativos e a unidade familiar, aprofundando aspectos tais como: religião, renda familiar, escolaridade, entre outros, sendo deste modo subsídio para a reorganização do cuidado na reestruturação do processo de desospitalização, pautado no perfil do usuário e da família em que este se encontra inserido.

\section{AGREDECIMENTOS:}

Escola Superior de Enfermagem de Coimbra e Hospital Federal Cardoso Fontes e a Coordenação de Aperfeiçoamento de Pessoal de Nível Superior - CAPES - Processo: Programa de Doutorado Sanduíche no Exterior -88881.132710/2016-01 e Ministério da Saúde. 


\section{REFERÊNCIAS}

1.Feuerwerker LCM, Merhy EE. A contribuição da atenção domiciliar para a configuração de redes substitutivas de saúde: desinstitucionalização e transformação de práticas. Pan Am J Public Health. [Internet] 2008;24(3) [acessoem 02 abr 2017]. Disponível: https://www.scielosp.org/pdf/rpsp/v24n3/a04v24n3.pdf.

2. World Health Organization (WHO). Expert Comminitte. World cancer report 2014.[Internet] WHO; 2014 [acesso em 02 abr 2017].Disponível: www.who.int/cancer/publications/WRC_2014/en/.

3. World Health Organization (WHO). Global Atlas of Palliative Care at the End of Life.[Internet] WHO; 2014 [acesso em 02 abr 2017]. Disponível: http://www.who.int/nmh/Global_Atlas_of_Palliative_Care.pdf.

4.da Silva MM, Buscher A, Moreira MC, Durte SCM. Visitando hospices na Alemanha e no Reino Unido na perspectiva dos cuidados paliativos.Esc Anna Nery Rev Enferm. [Internet] 2015;19(2) [acesso em 07mai2017]. Disponível:http:// dx.doi.org/10.5935/1414-8145.20150051.

5. Ministério da Saúde (BR). Secretaria de atenção básica. Departamento de Atenção Básica. Caderno de atenção domiciliar. [Internet] Brasília: Ministério da Saúde; 2012 [acesso em 02 abr 2017]. Disponível: http://bvsms.saude.gov.br/ bvs/publicacoes/caderno_atencao_domiciliar_melhor_casa.pdf.

6. Tigmanshu B. Palliative home care: a designer's perspective. Indian J. Palliative Care. [Internet] 2015;21(2) [acessoem01 jun 2017]. Disponível: http://dx.doi.org/10.4103/0973-1075.156512.

7. Meier DE, Beresford L. Palliative Care's Challenge: Facilitating Transitions of Care. JournalofPalliative Medicine. [Internet] 2008;11(3) [acesso em 02 abr 2017]. Disponível: http://dx.doi.org/10.1089/jpm.2008.9956.

8. Ministério da Saúde. Portaria n. 825, de 25 de abril de 2016. Redefine a Atenção Domiciliar no âmbito do Sistema Único de Saúde (SUS) e atualiza as equipes habilitadas. DiárioOficial da União. 2016 abr 25; Seção 1:44.

9. Coyle N. Palliative care, hospice care and bioethics: a natural fit. Journal of hospice \& palliative nursing. [Internet] 2014;16(1) [acessoem15 jun 2017] Disponível: http://dx.doi.org/10.1097/NJH.0000000000000032.

10. Ministério da Saúde. Portaria n. 874, de 16 de maio de 2013. Institui a Política Nacional para Prevenção e Controle do Câncer na Rede de Atenção à Saúde das Pessoas com Doenças Crônicas no Âmbito do SUS. Diário Oficial da União. 2013 mai 16.

11. Hui D, Cruz M, Mori M, Parsons H, Kwon J, Torres-Vigil I, et al. Concepts and definitions for "supportive care," "best supportive care," "palliative care," and "hospice care" in the published literature, dictionaries, and textbooks. SupportCareCancer. [Internet] 2013;21(3) [acesso em 15 jun 2017]. Disponível: http://dx.doi.org/10.1007/s00520-0121564-y.

12. daSilva KS, Kruse MHL. Em defesa da sociedade: a invenção dos cuidados paliativos e os dispositivos de segurança. Texto contexto - enferm. [Internet] 2013;22(2) [acesso em 05 jun 2017]. Disponível: http://dx.doi.org/10.1590/S010407072013000200030 .

13. Ministério da Saúde (BR). Conselho Nacional de Saúde. Diretrizes e normas regulamentadoras de pesquisa envolvendo seres humanos. Resolução n. 466, de 12 de dezembro de 2012. Brasília; 2012.

14. Brustolin A, Ferreti F. Itinerário terapêutico de idosos sobreviventes ao câncer. Acta paul. enferm. [Internet] 2017;30(1) [acesso em 00 mês 0000]. Disponível: http://dx.doi.org/10.1590/1982-0194201700008.

15. Moreira IMB. Competências do familiar cuidador da pessoa com doença oncológica em quimioterapia [tese]. Lisboa (PT): UniversidadeCatólica Portuguesa; 2013.

16. Reinhard SC, Feinberg LF, Choula, Houser A. Valuing the inualuable: 2015 up date. Undeniable progress, but big gaps remain. AARP Public Policy Institute. [Internet] 2015 [acesso em 01 jul 2017]. Disponível: https://www.aarp.org/ ppi/info-2015/valuing-the-invaluable-2015-update.html. 
17. Ferreira MLA, Pereira AMNA. Cuidar da pessoa com doença avançada na comunidade: Estudo fenomenológico. Rev. Enf. Ref. [Internet] 2016;4(8) [acesso em 01jul 2017]. Disponível: http://dx.doi.org/10.12707/RIV15029.

18. Ministério da Saúde (BR). Instituto Nacional de Câncer. Estimativa 2016: incidência de câncer no Brasil; 2016. [Internet] Brasilia: Ministério da Saúde; 2016 [acesso em 25 de maio de 2017]. Disponível: http://www.inca.gov.br/ bvscontrolecancer/publicacoes/edicao/Estimativa_2016.pdf.

19. Veras R. Envelhecimento populacional contemporâneo: demandas, desafios e inovações. Rev. Saúde Pública. [Internet] 2009;43(3) [acesso em 20 jun 2017]. Disponível: http://dx.doi.org/10.1590/S0034-89102009005000025.

20. Veras RP. Prevenção de doenças em idosos: os equívocos dos atuais modelos. Caderno de Saúde Pública. [Internet] 2012;28(10) [acesso em 25 mai 2017]. Disponível: http://dx.doi.org/10.1590/S0102-311X2012001000003.

21. Silva MS, Barbosa e Silva N, Alves AGP, Araújo SP, de Oliveira. Risco de doenças crônicas não transmissíveis na população atendida em Programa de Educação Nutricional em Goiânia (GO), Brasil. Ciênc. saúde coletiva. [Intenet]2014;19(5) [acesso em 05 jun 2017]. Disponível: http://dx.doi.org/10.1590/1413-81232014195.16312013.

22. Pontalti G, Rodrigues ESA, Firmino F, Fabris M, Stein MR, Longaray VK. Via subcutânea: Segunda opção em cuidados paliativos. Rev HCPA. [Internet] 2012;32(2) [acesso em 05jun2017]. Disponível: http://seer.ufrgs.br/index.php/hcpa/ article/view/26270.

23. Ferreira MAL, Pereira AMNA, Martins JCA, Barbieri-Figueiredo MC. Cuidados paliativos e enfermagem nas dissertações e teses em Portugal: um estudo bibliométrico. Rev. esc. enferm. USP. [Internet] 2016;50(2) [acesso em 01 jul 2017]. Disponível: http://dx.doi.org/10.1590/S0080-623420160000200019. 